\title{
Inhalation of carbon monoxide following resuscitation ameliorates hemorrhagic shock-induced lung injury
}

\author{
SUSUMU KAWANISHI ${ }^{1}$, TORU TAKAHASHI ${ }^{1,2}$, HIROSHI MORIMATSU ${ }^{1}$, HIROKO SHIMIZU $^{1}$, EMIKO OMORI ${ }^{1}$, \\ KENJI SATO $^{1}$, MASAKI MATSUMI ${ }^{1}$, SHIGERU MAEDA ${ }^{3}$, ATSUNORI NAKAO ${ }^{4}$ and KIYOSHI MORITA ${ }^{1}$ \\ ${ }^{1}$ Department of Anesthesiology and Resuscitology, Okayama University Medical School, Okayama 700-8558; \\ ${ }^{2}$ Faculty of Health and Welfare Science, Okayama Prefectural University, Okayama 719-1197; \\ ${ }^{3}$ Department of Dental Anesthesiology, Okayama University Dental School, Okayama 700-8558; \\ ${ }^{4}$ Department of Emergency and Critical Care Medicine, Hyogo College of Medicine, Nishinomiya, Hyogo 663-8501, Japan
}

Received June 28, 2012; Accepted October 30, 2012

DOI: $10.3892 / \mathrm{mmr} .2012 .1173$

\begin{abstract}
Even after successful resuscitation, hemorrhagic shock frequently causes pulmonary inflammation that induces acute lung injury (ALI). We previously demonstrated that when $\mathrm{CO}$ is inhaled at a low concentration both prior to and following hemorrhagic shock and resuscitation (HSR) it ameliorates HSR-induced ALI in rats due to its anti-inflammatory effects. In the present study, we administered $\mathrm{CO}$ to the same model of ALI only after resuscitation and examined whether it exerted a therapeutic effect without adverse events on HSR-induced ALI, since treatment of animals with CO prior to HSR did not prevent lung injury. HSR were induced by bleeding animals to achieve a mean arterial pressure of $30 \mathrm{mmHg}$ for $1 \mathrm{~h}$ followed by resuscitation with the removed blood. HSR resulted in the upregulation of inflammatory gene expression and increased the rate of apoptotic cell death in the lungs. This was determined from an observed increase in the number of cells positive for transferase-mediated dUTP-fluorescein isothiocyanate (FITC), nick-end labeling
\end{abstract}

Correspondence to: Dr Hiroshi Morimatsu, Department of Anesthesiology and Resuscitology, Okayama University Medical School, 251 Shikata-cho, Okayama 700-8558, Japan

E-mail: morima-h@md.okayama-u.ac.jp

Abbreviations: ALI, acute lung injury; CO, carbon monoxide; $\mathrm{COHb}$, carboxyhemoglobin; FITC, fluorescein isothiocyanate; HS, hemorrhagic shock; HSR, hemorrhagic shock and resuscitation; IgG, immunoglobulin G; IL-10, interleukin-10; iNOS, inducible nitric oxide synthase; LPS, lipopolysaccharide; MOF, multiple organ failure; mRNA, messenger ribonucleic acid; $\mathrm{PCO}_{2}$, partial pressure of carbon dioxide; $\mathrm{PO}_{2}$, partial pressure of oxygen; PPAR, peroxisome proliferator-activated receptor; RT-PCR, reverse transcription-polymerase chain reaction; TNF, tumor necrosis factor; TUNEL, terminal deoxynucleotidyl transferase-mediated dUTP-FITC nick-end labeling

Key words: acute lung injury, hemorrhagic shock, carbon monoxide, inflammation, peroxisome proliferator-activated receptor- $\gamma$ staining and activated caspase-3. HSR also resulted in prominent histopathological damage, including congestion, edema, cellular infiltration and hemorrhage. By contrast, $\mathrm{CO}$ inhalation for $3 \mathrm{~h}$ following resuscitation significantly ameliorated these inflammatory events, demonstrated by reduced histological damage, inflammatory mediators and apoptotic cell death. The protective effects of $\mathrm{CO}$ against lung injury were notably associated with an increase in the protein expression level of peroxisome proliferator-activated receptor (PPAR) $-\gamma$, an anti-inflammatory transcriptional regulator in the lung. Moreover, $\mathrm{CO}$ inhalation did not affect the hemodynamic status or tissue oxygenation during HSR. These findings suggest that inhalation of $\mathrm{CO}$ at a low concentration exerts a potent therapeutic effect against HSR-induced ALI and attenuates the inflammatory cascade by increasing PPAR- $\gamma$ protein expression.

\section{Introduction}

Hemorrhagic shock (HS), which is typically observed following traumatic and surgical injuries, leads to organ hypoperfusion, ischemia and additional reperfusion injuries during resuscitation. HS and resuscitation (HSR) frequently induces systemic inflammatory responses that result in lung inflammation (the lung is the first targeted organ). Advanced phase HSR leads to acute lung injury (ALI), which is responsible for the substantial morbidity and mortality in critically ill patients $(1,2)$. Pathogenesis is characterized by increased production of pro-inflammatory mediators and the activation of pulmonary inflammatory cells, which leads to development of interstitial edema and impaired lung function $(3,4)$. Therefore, modulation of these inflammatory responses may provide an important avenue for therapeutic intervention when treating lung injuries, including HSR-induced ALI.

Carbon monoxide (CO) is commonly considered poisonous in high concentrations due to its ability to interfere with oxygen delivery. However, $\mathrm{CO}$ is produced by the enzymatic reaction of heme oxygenase-1 (a stress-inducible protein) in the body and previous evidence indicates that endogenous $\mathrm{CO}$ is important in the physiological functioning of organs and possesses anti-inflammatory properties that have applications 
in a variety of biomedical fields (5). Moreover, administration of $\mathrm{CO}$ at low concentrations has been demonstrated to mediate potent cytoprotective and anti-inflammatory effects during hyperoxic lung injuries (6), endotoxemia (7), ischemic/reperfusion injuries (8) and HSR-induced multiple organ failure (MOF) (9). We previously demonstrated that inhalation of $\mathrm{CO}$ at low concentrations both prior to and following HSR exerts a potent protective effect against ALI in rats, as determined by improvements in histological findings as well as the suppression of the inflammatory signaling cascade (10). However, to evaluate the possible clinical applications of $\mathrm{CO}$ as a therapeutic modality, we must determine the protective effects of $\mathrm{CO}$ at various stages, including prior to or following resuscitation, as well as clarify the molecular mechanisms behind the anti-inflammatory effects of $\mathrm{CO}$.

In the present study, we have demonstrated that $\mathrm{CO}$ inhalation at a low concentration following resuscitation ameliorates HSR-induced ALI by attenuating apoptotic cell death in association with upregulation of peroxisome proliferator-activated receptor (PPAR)- $\gamma$, an anti-inflammatory transcriptional regulator. Present findings are likely to aid the expansion of new therapeutic avenues for $\mathrm{CO}$ gas in the field of therapeutic medical gas and critical care.

\section{Materials and methods}

Animals. The studies was approved by the Animal Use and Care Committee of the Okayama University Medical School (Okayama, Japan) in accordance with the guidelines for the care and use of laboratory animals that follows the ARRIVE reporting guidelines (11). Male Sprague-Dawley rats weighing 380-420 g (Clea Japan, Inc., Tokyo, Japan) were housed in a temperature-controlled $\left(25^{\circ} \mathrm{C}\right)$ room with a 12 -h light/dark cycle and free access to food and water until the start of the experiments.

HS protocol. All rats were subjected to sham or HS under pentobarbital anesthesia (sodium pentobarbital: $50 \mathrm{mg} / \mathrm{kg}$, i.p.) as previously described (12). Briefly, HS was initiated by withdrawing blood from the left femoral vein to achieve a mean arterial blood pressure of $30 \pm 5 \mathrm{mmHg}$ which was maintained for $1 \mathrm{~h}$. Arterial pressure was measured via catheters inserted into the left femoral artery. Rats were resuscitated with shed blood. The sham group underwent the same instrumentation procedures, with the exception of bleeding.

Experimental design. Rats were randomly assigned to the following three groups: sham-operated control (Sham), airtreated HSR (HSR/AIR) and CO-treated HSR (HSR/CO). $\mathrm{CO}$ exposure was performed at $250 \mathrm{ppm}$ for $3 \mathrm{~h}$ following the completion of resuscitation, as previously described (10). Rats in the Sham and HSR groups were maintained in room air in laminar flow cages. Some HSR rats were exposed to $\mathrm{CO}$ at $250 \mathrm{ppm}$ for $1 \mathrm{~h}$ prior to the onset of $\mathrm{HS}$ (CO/HSR). At specific time points following resuscitation, rats were sacrificed by decapitation under light anesthesia with ethyl ether. The lungs were excised and frozen immediately in liquid nitrogen and stored at $-80^{\circ} \mathrm{C}$ until further use.

In an additional set of experiments, HSR/AIR and HSR/CO rats were subjected to a median laparotomy under ether anes- thesia for $3 \mathrm{~h}$ following the onset of resuscitation, in order to examine the effect of $\mathrm{CO}$ exposure on the $\mathrm{CO}$ content of lung tissues. Blood was collected from the abdominal aorta and the lungs were perfused in situ with physiological saline until the venous effluent became clear. The lungs were then removed for preparation of tissue homogenates.

Histological study. Tissues fixed in $10 \%$ neutral-buffered formalin were embedded in paraffin and cut in 4-6 $\mu$ m-thick sections. After deparaffinization, the sections were stained with hematoxylin and eosin followed by a light microscope examination by a blinded observer. Grading of lung injury severity was performed according to previously described methods with modifications for five independent experiments (13-15).

Lung sections adjacent to those used for histopathological study were subjected to staining for neutrophils with a naphthol AS-D chloroacetate esterase-staining kit (Sigma Diagnostics, St. Louis, MO, USA) as described previously (16). The number of positively stained cells was counted in five nonconsecutive sections per rat at a x400 magnification by a blinded observer.

Lung wet weight to dry weight ratio. Lung tissue samples were blotted and weighed (wet weight) before drying for $24 \mathrm{~h}$ at $110^{\circ} \mathrm{C}$ (dry weight). The wet weight/dry weight ratio was calculated by dividing the wet weight by the dry weight as an index of pulmonary edema (17).

RNA isolation and northern blot analysis. Total RNA was isolated from the frozen rat tissues with Tri-Reagent ${ }^{\mathrm{TM}}$ (Sigma) according to the manufacturer's instructions. Northern blotting was performed as described previously (17). Briefly, $20 \mu \mathrm{g}$ of total RNA was separated on a $1.2 \%(\mathrm{w} / \mathrm{v})$ agarose gel containing $6.5 \%(\mathrm{v} / \mathrm{v})$ formaldehyde and transferred on Bio-Rad Zeta-Probe membrane (Bio-Rad Laboratories, Richmond, CA, USA). The blotted membrane was hybridized with $\left[\alpha{ }^{32} \mathrm{P}\right] \mathrm{dCTP}-$ labeled cDNA probes for tumor necrosis factor (TNF)- $\alpha$ and inducible nitric oxide synthase (iNOS), respectively, followed by washing at high stringency. The membrane was exposed to X-ray films at $-70^{\circ} \mathrm{C}$, and autoradiographs and $18 \mathrm{~S}$ ribosomal RNA were quantified with an image scanner (GelPrintTM 2000i, Genomic Solutions, Ann Arbor, MI, USA) and computerized image analysis software (Basic Quantifier ${ }^{\mathrm{TM}}$ version 3.0, Genomic Solutions). The relative amounts of radiolabeled cDNA that hybridized to the blots were normalized to $18 \mathrm{~S}$ ribosomal RNA levels for correction of loading errors.

Preparation of nuclear extracts and western blot analysis. Nuclear extracts were isolated from the lung tissues using Nuclear and Cytoplasmic Extraction Reagents (NE-PER ${ }^{\mathrm{TM}}$; Thermo Fisher Scientific, Inc., Rockford, IL, USA), as previously described (18). The nuclear protein content was determined using the BCA Protein Assay kit ${ }^{\mathrm{TM}}$ (Thermo Fisher Scientific, Inc.). Samples were analyzed on a $12.5 \%$ polyacrylamide gel (e-PAGEL ${ }^{\mathrm{TM}}$; ATTO, Tokyo, Japan), according to the manufacturer's instructions. Following electrophoresis, proteins were transferred to a Hybond-P PVDF membrane (GE Healthcare, Little Chalfont, UK) and blocked with 4\% (w/v) BlockAce ${ }^{\mathrm{TM}}$ (DS Pharma Biomedical Co., Ltd., 
Osaka, Japan). Blots were incubated with rabbit anti-PPAR- $\gamma$ polyclonal antibody (1:1,200; Abcam, Cambridge, UK) and then treated with horseradish peroxidase-labeled goat antirabbit immunoglobulin G (IgG; Santa Cruz Biotechnology, Inc., Santa Cruz, CA, USA). The antigen-antibody complexes were visualized using an ECL ${ }^{\mathrm{TM}}$ chemiluminescence system (GE Healthcare).

Real-time reverse transcription (RT)-polymerase chain reaction $(P C R)$. Real-time RT-PCR was performed using SYBR Premix Ex Taq ${ }^{\mathrm{TM}}$ (Takara Bio, Shiga, Japan) and a LightCycler (Roche Diagnostics GmbH, Mannheim, Germany), as previously described (16). The interleukin-10 (IL-10) messenger RNA (mRNA) level was normalized to the $\beta$-actin mRNA level.

Histological detection of apoptotic cell death. Detection and quantification of apoptotic cells were performed using the terminal deoxynucleotidyl transferase-mediated dUTP-fluorescein isothiocyanate (FITC) nick-end labeling (TUNEL) method with the MEBSTAIN Apoptosis kit II (MBL, Nagoya, Japan), according to the manufacturer's instructions. Sections were incubated with FITC-labeled avidin and then counterstained with $0.5 \mu \mathrm{g} / \mathrm{ml}$ propidium iodide to visualize the nuclei.

Immunostaining of activated caspase-3 was performed using rabbit polyclonal primary antibody (1:250; Asp 175, no. 9661; Cell Signaling Technology, Danvers, MA, USA), according to the manufacturer's instructions. Sections were incubated with diluted Cyanine 3-conjugated donkey anti-rabbit secondary antibody (1:100; Chemicon International, Temecula, CA, USA).

Apoptotic and activated caspase-3-positive cells were counted using a confocal laser scanning microscope (model LSM510; ZEISS, Jena, Germany).

Measurement of carboxyhemoglobin (COHb) and tissue $\mathrm{CO}$ content. Blood $\mathrm{COHb}$ levels in the arterial blood were measured using the OSM3 Hemoximeter (Radiometer Copenhagen, Copenhagen, Denmark). The tissue CO content was measured as previously described (10).

Detection of tissue hypoxia by pimonidazole adducts. Levels of tissue hypoxia were detected by pimonidazole hydrochloride staining on lung (Hypoxyprobe ${ }^{\mathrm{TM}}-1$, Natural Pharmacia International, Inc., Burlington, MA, USA) as described previously (19). Slides were stained with a primary mouse monoclonal antibody for pimonidazole adducts, followed by a secondary FITC donkey anti-mouse IgG (Chemicon International). Images were captured as described earlier. Hypoxic rats breathed 6\% oxygen continuously for $90 \mathrm{~min}$ as positive control. The atmosphere in the cages was sampled continuously from the chamber exhaust outlet and the $\mathrm{O}_{2}$ concentration was monitored with a gas analyzer (CAPNOMAC $^{\mathrm{TM}}$, Datex-Ohmeda, Finland).

Statistical analysis. Statistical analysis was undertaken using the Student's t-test or analysis of variance followed by Scheffé's F-test, as appropriate. $\mathrm{P}<0.05$ was considered to indicate a statistically significant result. Data are shown as the mean \pm SEM. We used statistical package Statview (Abacus Concepts, Berkeley, CA, USA).

\section{Results}

Therapeutic effect of CO inhalation on HSR-induced lung injury. While sections obtained from the Sham rats appeared normal, sections from HSR rats developed interstitial edema, as demonstrated by pronounced alveolar septal thickening and inflammatory cell infiltration $12 \mathrm{~h}$ after HSR (Fig. 1). CO exposure for $3 \mathrm{~h}$ following HSR markedly mitigated these pathological changes, including congestion, edema, inflammation and hemorrhage; however, $\mathrm{CO}$ exposure for $1 \mathrm{~h}$ prior to HSR did not affect the pathological findings (Fig. 1A). The significant effects of $\mathrm{CO}$ exposure following HSR were also confirmed by the scoring system based on histopathological changes reviewed by a blinded independent researcher. Only $\mathrm{CO}$ inhalation following HSR suppressed lung injuries, as revealed by histological damage that was confirmed by a decrease in the total histological score (Fig. 1B). By contrast, CO inhalation prior to HSR did not affect the histopathological scores (Fig. 1C).

Effect of $\mathrm{CO}$ inhalation on neutrophil sequestration in lungs following HSR. The number of infiltrating neutrophils was markedly increased in the HSR/AIR group at $12 \mathrm{~h}$ following resuscitation compared with that in the Sham group (Fig. 2A and B). By contrast, neutrophil recruitment was identified as significantly reduced in the lungs of the $\mathrm{CO}$-treated rats (Fig. 2A and B).

CO inhalation reduced lung edema following HSR. Lung wet/dry ratio, a parameter of lung edema, was identified as significantly increased within $12 \mathrm{~h}$ in the HSR group, compared with that of the Sham group (Fig. 2C). However, CO inhalation following HSR significantly attenuated HSR-induced lung edema (Fig. 2C).

Effect of $C O$ treatment on the gene expression of HSR-induced inflammatory mediators, including TNF- $\alpha$ and iNOS. Although mRNA levels of TNF- $\alpha$ and iNOS were barely detectable in the Sham group, these genes were significantly upregulated in the HSR/AIR group at $3 \mathrm{~h}$ after resuscitation (Fig. 3). CO inhalation following HSR significantly decreased mRNA expression levels of TNF- $\alpha$ and iNOS to almost the same levels as those measured in the Sham group (Fig. 3).

Effect of CO treatment on the expression of IL-10 and PPAR- $\gamma$ in the lungs following HSR. Pulmonary IL-10 mRNA was barely detectable in the Sham group (Fig. 4A). The HSR procedure slightly increased the IL-10 mRNA level in the HSR/AIR group compared with that in the Sham group (Fig. 4A). Notably, CO treatment resulted in further upregulation of IL-10 mRNA (Fig. 4A). While PPAR- $\gamma$ protein was expressed at high levels in the Sham group, levels in the HSR/AIR group was almost the same as that in the Sham group at $3 \mathrm{~h}$ following resuscitation (Fig. 4B and C). However, CO treatment significantly increased this level in comparison to that measured in the HSR/AIR group (Fig. 4B and C). The level measured in the HSR/CO group was almost twice as large as that measured in the HSR/AIR group (Fig. 4B and C). 

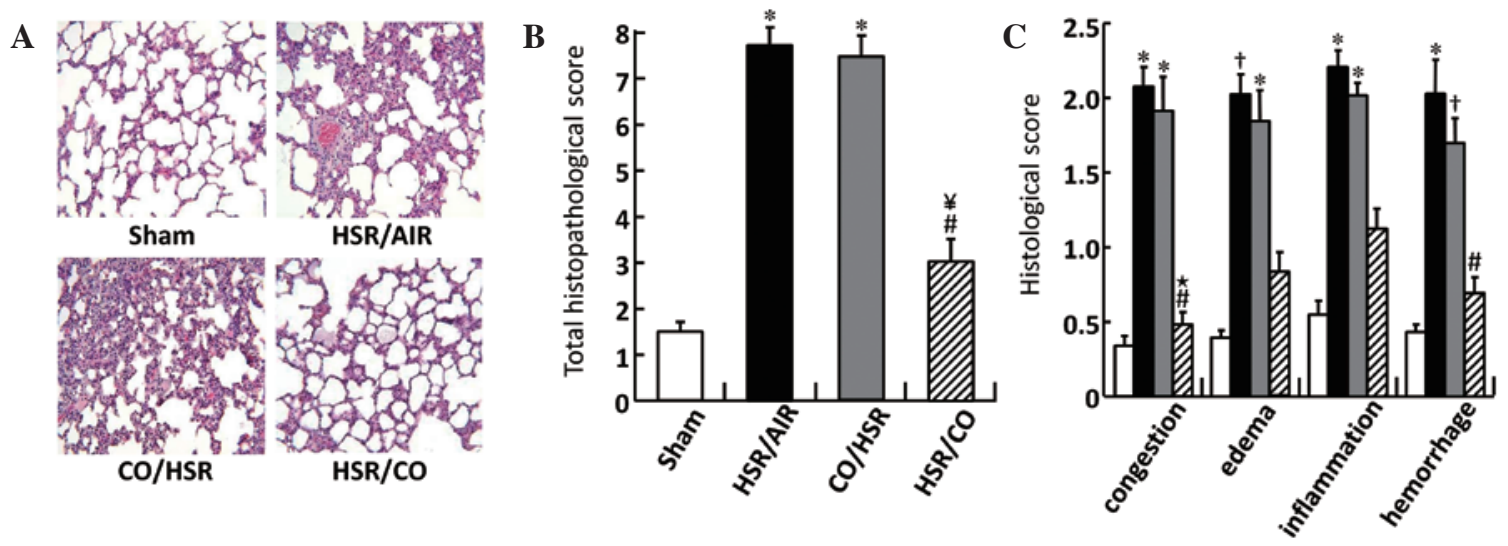

Figure 1. Comparative effects of carbon monoxide $(\mathrm{CO})$ exposure at various times on histological changes in the lungs following hemorrhagic shock and resuscitation (HSR). (A) Rats subjected to HSR were exposed to $\mathrm{CO}$ for $1 \mathrm{~h}$ prior to onset of hemorrhagic shock or for $3 \mathrm{~h}$ following resuscitation or were maintained in air. Lungs from HSR rats following these various treatments were excised $12 \mathrm{~h}$ after resuscitation and subjected to histological examination. Representative images from five independent experiments (hematoxylin-eosin staining, x200 original magnification). (B) Severity of histopathological changes in the lungs were graded according to criteria for congestion, edema, inflammation and hemorrhage. Ten areas of the lung parenchyma from each rat were graded as 0 (no findings or normal), 1 (mild), 2 (moderate) or 3 (severe) for each of the four parameters. (C) Sum of histological scores for each of the four parameters were calculated ( $\mathrm{n}=5$, for each group). ${ }^{\mathrm{P}} \mathrm{P}<0.01$ vs. Sham; ${ }^{ } \mathrm{P}<0.05$ vs. Sham; ${ }^{*} \mathrm{P}<0.01$ vs. HSR/AIR; ${ }^{\mathrm{q}} \mathrm{P}<0.01$ vs. CO/HSR. Sham, sham-operated control rats; HSR/AIR, HSR rats maintained in air; CO/HSR, HSR rats exposed to CO for $1 \mathrm{~h}$ prior to hemorrhagic shock; HSR/CO, HSR rats exposed to CO for $3 \mathrm{~h}$ following resuscitation

A
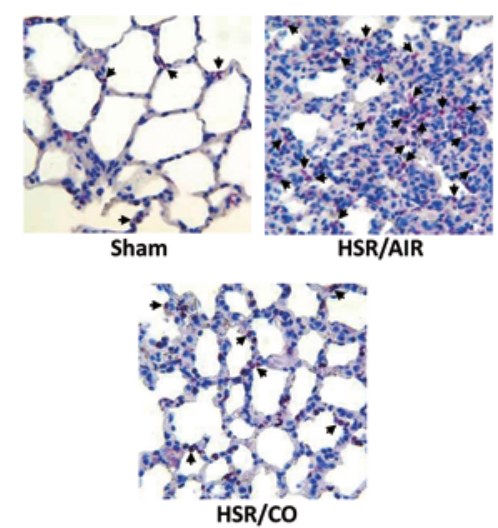

B

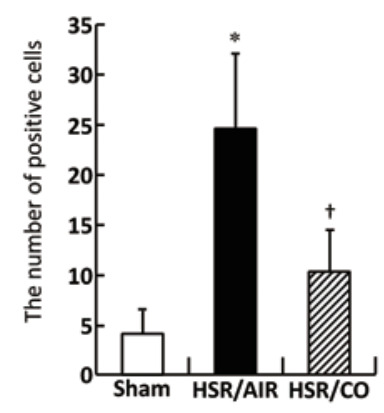

C

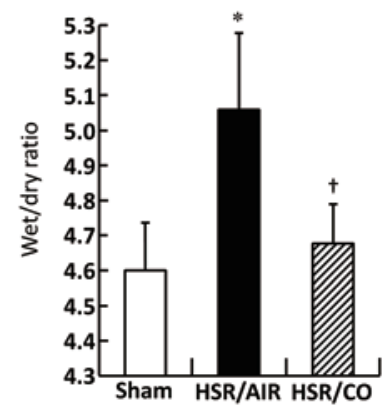

Figure 2. Effect of carbon monoxide (CO) inhalation on neutrophil accumulation in the lungs and wet/dry-weight (W/D) ratio of the lungs following hemorrhagic shock and resuscitation (HSR). Rats subjected to HSR were exposed to CO for $3 \mathrm{~h}$ following resuscitation or maintained in air. Lungs were excised $12 \mathrm{~h}$ after resuscitation and were used for the staining of neutrophils using the naphthol AS-D chloroacetate method and the determination of the W/D ratio. (A) Representative images of five independent experiments. Arrows indicate positively stained neutrophils (x400 original magnification). (B) Number of neutrophils in the lung sections of five nonconsecutive sections/rat at $\mathrm{x} 400$ magnification ( $\mathrm{n}=5$ for each group). ${ }^{*} \mathrm{P}<0.01 \mathrm{vs.} \mathrm{Sham;}{ }^{\dagger} \mathrm{P}<0.01 \mathrm{vs}$. HSR/AIR. (C) W/D ratio as an index of pulmonary edema ( $\mathrm{n}=5$, for each group). " $\mathrm{P}<0.01$ vs. Sham; ${ }^{\dagger} \mathrm{P}<0.05$ vs. HSR/AIR. Sham, sham-operated control rats; HSR/AIR, air-treated HSR rats; HSR/CO, CO-treated HSR rats.

A

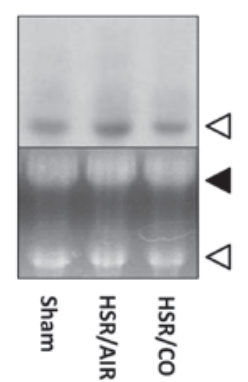

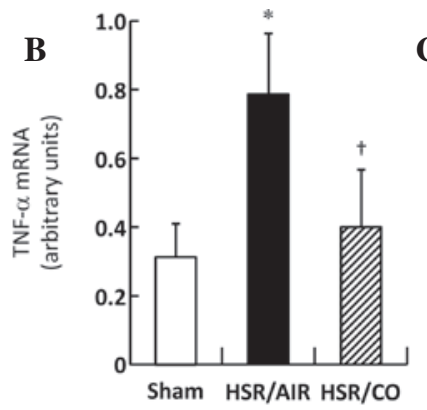

C

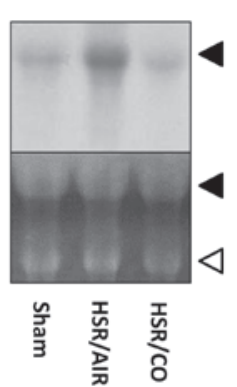

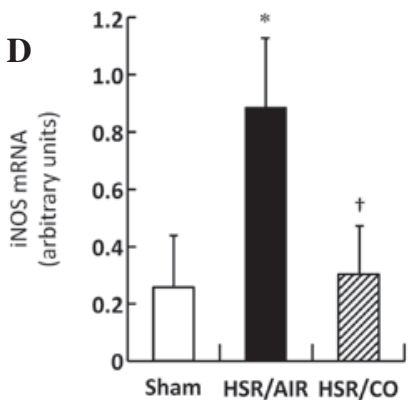

Figure 3. Effect of carbon monoxide (CO) inhalation on gene expression of tumor necrosis factor (TNF)- $\alpha$ and inducible nitric oxide synthase (iNOS) in the lungs following hemorrhagic shock and resuscitation (HSR). Lungs from HSR rats exposed to $\mathrm{CO}$ for $3 \mathrm{~h}$ following resuscitation or maintained in air were excised $3 \mathrm{~h}$ after resuscitation and expression levels of TNF- $\alpha$ and iNOS mRNA were determined by northern blot analysis. Autoradiographic signals of RNA blot hybridized with $\left[\alpha-{ }^{32} \mathrm{P}\right]$ dCTP-labeled (A) TNF- $\alpha$ or (C) iNOS cDNA. Closed and open arrowheads indicate 28S and 18S ribosomal RNA, respectively. Densitometric analysis of (B) TNF- $\alpha$ and (D) iNOS mRNA, normalized to 18S ribosomal RNA. Concentrations are expressed in arbitrary units. Data are presented as the mean \pm SEM ( $\mathrm{n}=5$, for each group). ${ }^{*} \mathrm{P}<0.01$ vs. Sham; ${ }^{\circ} \mathrm{P}<0.05$ vs. HSR/AIR. Sham, sham-operated control rats; HSR/AIR, air-treated HSR rats; $\mathrm{HSR} / \mathrm{CO}, \mathrm{CO}$-treated HSR rats. 
Table I. Effect of inhaled carbon monoxide (CO) on central hemodynamics following HSR.

\begin{tabular}{|c|c|c|c|c|c|c|c|}
\hline \multirow[b]{2}{*}{$\begin{array}{l}\text { Rat } \\
\text { treatment }\end{array}$} & \multicolumn{2}{|c|}{$\begin{array}{l}\text { Mean arterial pressure } \\
\qquad(\mathrm{mmHg})\end{array}$} & \multicolumn{2}{|c|}{ Heart rate (beats/min) } & \multicolumn{3}{|c|}{$\begin{array}{c}\text { Arterial blood gas } \\
\text { analysis ( } 3 \text { h after HSR) }\end{array}$} \\
\hline & $\begin{array}{l}\text { End of } \\
\text { resuscitation }\end{array}$ & $\begin{array}{l}3 \mathrm{~h} \text { after } \\
\text { HSR }\end{array}$ & $\begin{array}{l}\text { End of } \\
\text { resuscitation }\end{array}$ & $\begin{array}{l}3 \mathrm{~h} \text { after } \\
\text { HSR }\end{array}$ & $\mathrm{pH}$ & $\begin{array}{c}\mathrm{PO}_{2} \\
(\mathrm{mmHg})\end{array}$ & $\begin{array}{c}\mathrm{PCO}_{2} \\
(\mathrm{mmHg})\end{array}$ \\
\hline HSR/AIR & $100.0 \pm 3.03$ & $70.0 \pm 5.76$ & $315.2 \pm 4.08$ & $344.0 \pm 21.76$ & $7.412 \pm 0.013$ & $101.4 \pm 5.96$ & $44.8 \pm 1.80$ \\
\hline $\mathrm{HSR} / \mathrm{CO}$ & $100.4 \pm 10.97$ & $73.2 \pm 8.38$ & $284.8 \pm 18.00$ & $297.6 \pm 5.31$ & $7.412 \pm 0.013$ & $117.0 \pm 12.95$ & $40.7 \pm 1.36$ \\
\hline
\end{tabular}

Rats subjected to HSR were exposed to $\mathrm{CO}$ for $3 \mathrm{~h}$ following resuscitation or maintained in air. Mean arterial pressure and heart rate were measured at the completion of resuscitation and $3 \mathrm{~h}$ after resuscitation. Arterial blood gas analysis was performed $3 \mathrm{~h}$ after resuscitation. Data are presented as the mean \pm SEM ( $n=5$ for each group). HSR/AIR, air-treated HSR rats; HSR/CO, CO-treated HSR rats. HSR, hemorrhagic shock and resuscitation; $\mathrm{PO}_{2}$, partial pressure of oxygen; $\mathrm{PCO}_{2}$, partial pressure of carbon dioxide.

A

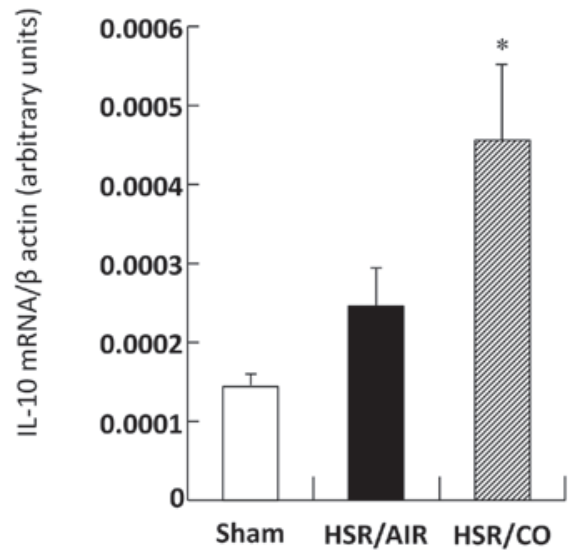

B

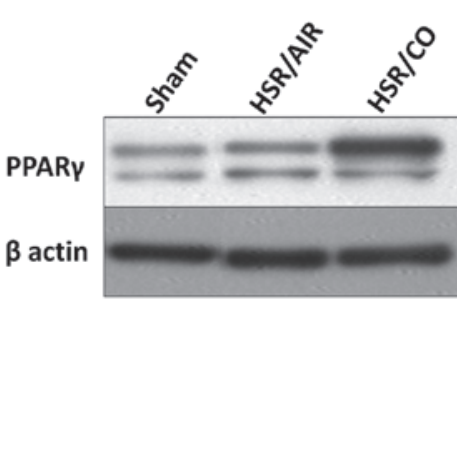

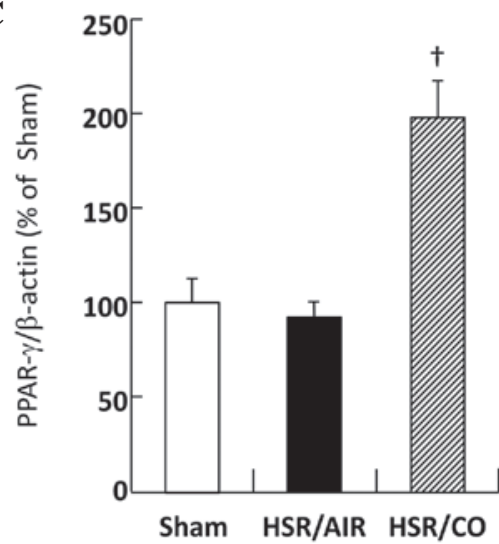

Figure 4. Effect of carbon monoxide (CO) inhalation on IL-10 gene expression and PPAR- $\gamma$ protein expression in the lungs following hemorrhagic shock and resuscitation (HSR). Lungs from HSR rats exposed to $\mathrm{CO}$ for $3 \mathrm{~h}$ following resuscitation or maintained in air were excised $3 \mathrm{~h}$ after resuscitation and levels of IL-10 mRNA and PPAR- $\gamma$ protein were determined by real-time RT-PCR and western blot analysis, respectively. (A) IL-10 mRNA level was normalized to $\beta$-actin and is expressed in arbitrary units. Data are presented as the mean \pm SEM ( $n=12$ for each group). * $\mathrm{P}<0.05$ vs. HSR/AIR. (B) Western blot analysis using anti-rat PPAR- $\gamma$ and anti- $\beta$-actin as the loading control. (C) Densitometric analysis of blot normalized to the density of $\beta$-actin, PPAR- $\gamma$ is expressed as percentage concentration of the Sham group. Data are presented as the mean \pm SEM ( $\mathrm{n}=10$ for each group). ${ }^{\dagger} \mathrm{P}<0.01 \mathrm{vs}$. HSR/AIR. Sham, sham-operated control rats; HSR/AIR, air-treated HSR rats; HSR/CO, CO-treated HSR rats.

Effect of $C O$ inhalation on apoptotic cell death in the lungs. While TUNEL-positive cells were barely detected in the Sham group, their numbers were significantly increased at $12 \mathrm{~h}$ following HSR in the lungs of the experimental rats (Fig. 5A and $\mathrm{B})$. By contrast, $\mathrm{CO}$ inhalation significantly decreased the number by $\sim 30 \%$ of the level measured in the HSR/AIR group (Fig. 5A and B). Activated caspase-3-positive cells were barely detected in the lungs of the Sham group, while their number was significantly increased at $12 \mathrm{~h}$ following resuscitation in the HSR/AIR rats (Fig. 5C and D). By contrast, the number of activated caspase-3-positive cells in the CO-treated HSR rats was low low $(\mathrm{P}<0.027$; HSR/AIR vs. HSR/CO) and at almost the same level as that measured in the Sham group (Fig. 5C and D).

Effect of $\mathrm{CO}$ inhalation on blood $\mathrm{COHb}$ levels and $\mathrm{CO}$ tissue content in the lungs. Blood COHb levels in the HSR/CO group were markedly increased $(19.40 \pm 0.66 \%)$ compared with those in the HSR/AIR group (3.08 $\pm 0.06 \%$; Fig. $6 \mathrm{~A})$. CO content in the lungs was markedly increased to $5.43 \pm 0.89 \mathrm{pmol} / \mathrm{mg}$ tissue following $\mathrm{CO}$ exposure $3 \mathrm{~h}$ after resuscitation, while $\mathrm{CO}$ content in the lungs of the HSR/AIR group was $0.49 \pm 0.02 \mathrm{pmol} / \mathrm{mg}$ tissue (Fig. 6B).

CO did not affect the hemodynamic status during HSR. Mean arterial pressure and heart rate in the HSR/CO group were almost identical to those in the HSR/AIR group, even at $3 \mathrm{~h}$ after resuscitation (Table I). Moreover, $\mathrm{CO}$ exposure did not exert a significant effect on $\mathrm{pH}$, partial pressure of oxygen $\left(\mathrm{PO}_{2}\right)$ and partial pressure of carbon dioxide $\left(\mathrm{PCO}_{2}\right)$ levels in the arterial blood (Table I).

CO did not induce tissue hypoxia. While there was no pimonidazole-binding in sham-operated control rats, sections from unoperated animals exposed to hypoxia, used as positive controls, revealed significantly enhanced pimonidazole-binding compared with sham-operated control rats (Fig. 7). By contrast, sections from HSR rats $3 \mathrm{~h}$ after resuscitation revealed no pimonidazole-binding, regardless of CO inhalation (Fig. 7). 
A

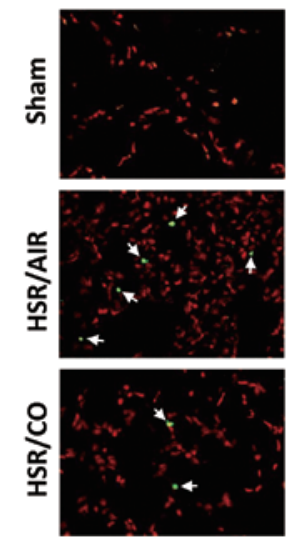

B

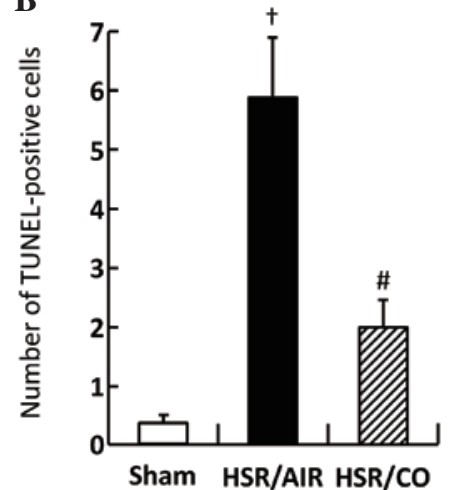

C

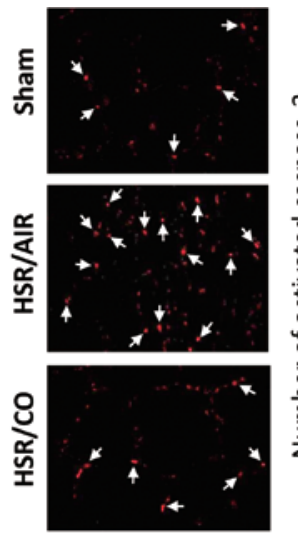

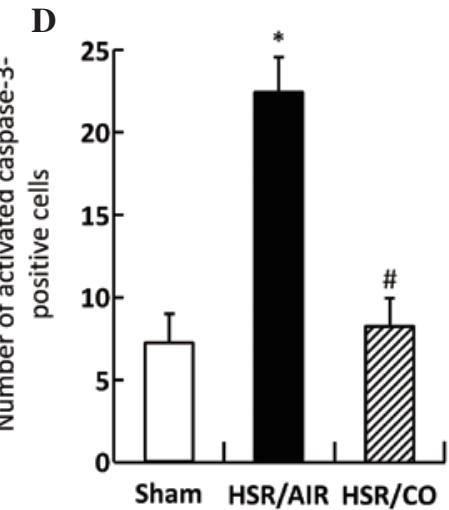

Figure 5. Effect of carbon monoxide (CO) inhalation on apoptotic cell death in the lungs following hemorrhagic shock and resuscitation (HSR). Lungs from HSR rats exposed to $\mathrm{CO}$ for $3 \mathrm{~h}$ following resuscitation or maintained in air were excised $12 \mathrm{~h}$ after resuscitation. (A) Sections were stained by TUNEL method (representative images of five independent experiments) and (C) immunohistochemical analysis of activated caspase-3. The arrows indicate TUNEL- or activated caspase-3-positive cells (x400 original magnification). Quantification of (B) TUNEL- and (D) activated caspase-3-positive cells (n=5 for each group). ${ }^{*} \mathrm{P}<0.05$ vs. Sham; ${ }^{\dagger} \mathrm{P}<0.01$ vs. Sham; ${ }^{*} \mathrm{P}<0.05$ vs. HSR/AIR. Sham, sham-operated control rats; HSR/AIR, air-treated HSR rats; HSR/CO, CO-treated HSR rats TUNEL, terminal deoxynucleotidyl transferase-mediated dUTP-FITC nick-end labeling.
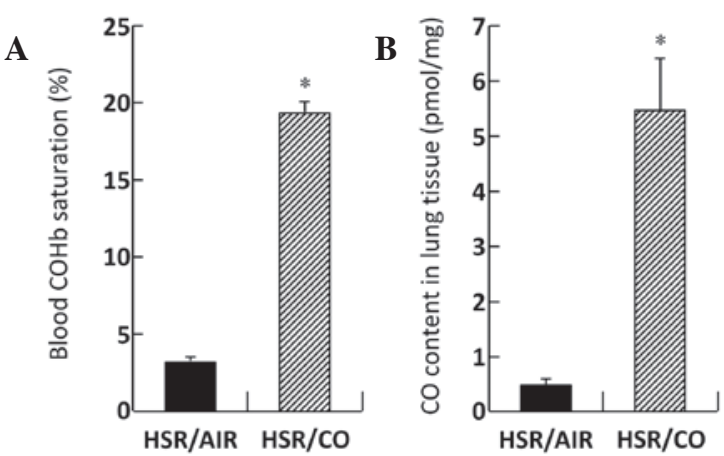

Figure 6. Effect of carbon monoxide (CO) inhalation on blood CO-hemoglobin $(\mathrm{COHb})$ levels and $\mathrm{CO}$ tissue content in the lungs following hemorrhagic shock and resuscitation (HSR). HSR rats were exposed to CO for $3 \mathrm{~h}$ following resuscitation or maintained in air. Three hours after resuscitation, (A) $\mathrm{COHb}$ levels in the arterial blood and (B) $\mathrm{CO}$ content in the lungs were measured. Data are presented as the mean \pm SEM ( $n=5$ for each group). " $\mathrm{p}<0.01$ vs. HSR/AIR. HSR/AIR, air-treated HSR rats; HSR/CO, CO-treated HSR rats.

\section{Discussion}

The present study demonstrates that inhalation of $\mathrm{CO}$ at a low concentration following HSR exerts potent therapeutic effects against HSR-induced ALI. The findings of this study indicate that $\mathrm{CO}$ treatment suppresses molecular activation of HSR-induced inflammatory events. CO exerted anti-inflammatory effects that are, at least in part, mediated through induction of IL-10, an anti-inflammatory cytokine and activation of PPAR- $\gamma$, an anti-inflammatory transcriptional regulator. HS leads to exaggerated systemic inflammatory responses, which may be important in the development of MOF. MOF is associated with high mortality rates, reported in specific cases to exceed $50 \%$ (1). ALI is the first clinical manifestation of organ failure and a major factor leading to MOF, which is characterized by acute lung inflammation involving the local recruitment and activation of polymorphonuclear leukocytes and the release of pro-inflammatory mediators $(1,20)$. Therefore, a novel anti-inflammatory modality that attenuates
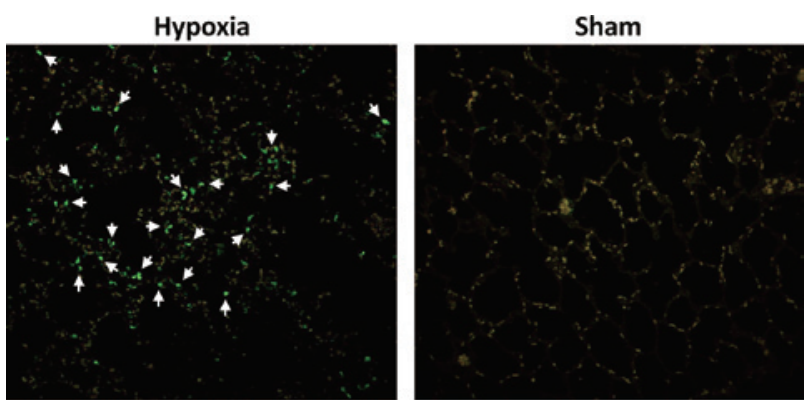

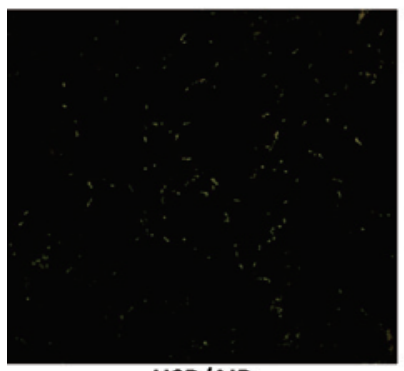

HSR/AIR

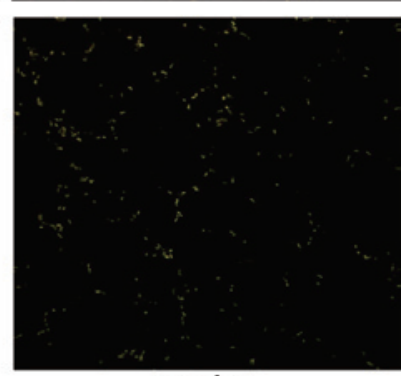

$\mathrm{HSR} / \mathrm{CO}$
Figure 7. Detection of tissue hypoxia with hypoxia marker pimonidazole. Rats pretreated with hypoxia marker pimonidazole were subjected to HSR. Following resuscitation, HSR rats were exposed to $\mathrm{CO}$ for $3 \mathrm{~h}$ or maintained in air. Lungs were excised $3 \mathrm{~h}$ after HSR and pimonidazole binding cells were detected. Representative images from five independent experiments are shown. Arrows indicate hypoxic cells binding with pimonidazole (x200, original magnification). Hypoxia, rats exposed to $6 \%$ oxygen for $1.5 \mathrm{~h}$ used as hypoxic control; Sham, air-treated rats with sham surgery; HSR/AIR, airtreated HSR rats; HSR/CO, CO-treated HSR rats.

the overwhelming lung inflammation that develops following HS-induced lung injury may hold important therapeutic potential.

We previously demonstrated that combined treatment of $\mathrm{CO}$ prior to and following HSR exerts potent anti-inflammatory effects, resulting in reduced inflammatory cell influx into the lungs and marked attenuation in the expression of proinflammatory cytokines (10). The present study demonstrates that exposure to $\mathrm{CO}$ alone following HSR confers cytoprotective effects on HSR-induced lung injuries, primarily through 
its anti-inflammatory effects. This finding suggests that post-treatment use of $\mathrm{CO}$ may have potential as a therapeutic approach against lung injuries and inflammation that result from multiple causes. Prevention of lung injuries by treatment with CO prior to noxious stimuli, including lipopolysaccharide (LPS), hyperoxia and ischemia/reperfusion, have been described by other investigators using experimental animal models $(6,7,21)$. The results of these studies are consistent with our findings, but treatment with $\mathrm{CO}$ following harmful insult also has been reported to provide protection against lung injuries that are induced by various oxidative stimuli (22-25). Novel observations of the present study provide further evidence that post-treatment use of $\mathrm{CO}$ represents a potent therapeutic modality for treatment of ALI, which may contribute to clinical benefits in the treatment of lung injuries and inflammation.

$\mathrm{CO}$ possesses various anti-inflammatory properties that contribute to the reduction of oxidative stress and apoptotic cell death and are, at least in part, mediated through the p38 mitogen-activated protein kinase signaling pathway (26). However, the exact molecular mechanisms of anti-inflammatory actions of $\mathrm{CO}$ remain elusive. Previous studies, including ours, indicate that $\mathrm{CO}$ inhibits the activation of nuclear factor $\kappa \mathrm{B}$ and activator protein-1, leading to amelioration of inflammatory lung disease by various causes, including LPS and HSR $(10,27,28)$. The beneficial effects of CO have also been reported in association with the upregulation of IL-10 expression (26). Moreover, a previous study indicated that the anti-inflammatory effects of CO involve the inhibition of upstream toll-like receptor signaling pathways (29). Thus, multiple mechanistic actions of CO have been postulated. Previous observations have suggested another mechanism of $\mathrm{CO}$ that is exerted on the inflammatory response, which is mediated by PPAR- $\gamma(30,31)$. In accordance with these reports, upregulation of PPAR- $\gamma$ was accompanied by the protective effects of CO against ALI following HSR in the present study. Previous observations clearly demonstrate that the endogenous ligands of PPAR $-\gamma$ reduce organ injury and dysfunction, including detrimental effects to the liver, kidneys, intestines and lungs, which are caused by HS (32). More recently, augmented PPAR $-\gamma$ expression by exogenous agonists was demonstrated to contribute to the modulation of systemic and pulmonary inflammation in HSR (33). These investigators also indicated that PPAR- $\gamma$ agonists exert anti-apoptotic effects in the lungs following severe hemorrhage (34). Further studies using PPAR- $\gamma$-neutralizing antibodies or PPAR- $\gamma$-knockout animals are likely to be important in clarification of the function of PPAR- $\gamma$ in HSR.

Although it is important to establish a clinically applicable strategy for using $\mathrm{CO}$ in treatment of ALI following HSR, CO exposure is associated with serious health problems. CO is well known for its high affinity for hemoglobin, which is more than 200 times greater than that of oxygen, as well as its ability to block the binding of oxygen (35), thereby leading to impaired delivery of oxygen to tissues and ultimately resulting in hypoxic tissue damage and death (36). In the present study, rats exposed to $250 \mathrm{ppm}$ $\mathrm{CO}$ for $3 \mathrm{~h}$ exhibited considerably high blood $\mathrm{COHb}$ levels $(19.40 \pm 0.66 \%)$. However, arterial blood gas analysis revealed that there was no difference in $\mathrm{PaO}_{2}$ levels between $\mathrm{CO}$ and
AIR treated-rats in the HSR groups (Table I). Moreover, as shown in Fig. 7, CO inhalation did not induce lung tissue hypoxia as determined by the pomonidazole hydrochloride staining, which is known as an in vivo hypoxia marker (19). Instead, $\mathrm{CO}$ treatment of rats markedly improved the clinical presentation of HSR-induced ALI.

There are two concerns regarding species differences, particularly between small and large animals, prior to clinical application of $\mathrm{CO}$. One is the differences is rate of $\mathrm{CO}$ uptake into the body. Longer reaction time is required to form $\mathrm{COHb}$ in large animals, including humans, than in small animals, including mice and rats, which is attributable to the lower minute volume of ventilation/unit of body weight in large animals (37). The other is differences in affinity of CO to hemoglobin. The affinity of $\mathrm{CO}$ to hemoglobin is approximately 4 times higher than that of oxygen in rodents (38). The magnitude of the affinity differences between two gases is significantly smaller in rodents than in humans. Thus, humans may inhale higher concentration of $\mathrm{CO}$ than mice to obtain the same level of $\mathrm{CO}$ in the tissue which is released from $\mathrm{COHb}$. However, it was recently reported that $\mathrm{CO}$ inhalation only at 100 ppm suppresses LPS-induced pulmonary inflammation in mice (39). This allows us to hypothesize that $\mathrm{CO}$ exposure below the lethal toxicity dose may exert a protective effect on lung injury and inflammation in the clinical setting.

In conclusion, the present study demonstrates that HSR causes significant pulmonary inflammation, as shown by the increase in the gene expression levels of inflammatory mediators, neutrophil emigration and pulmonary edema, which leads to apoptotic cell death. CO inhalation following HSR significantly reduces these indices with additional increases in gene expression of IL-10, an anti-inflammatory cytokine and protein expression of PPAR- $\gamma$, an anti-inflammatory transcriptional regulator, resulting in amelioration of HSR-induced injury. These findings indicate that $\mathrm{CO}$ inhalation possesses a potent therapeutic effect on HSR-induced lung injury, at least in part, through the attenuation of the inflammatory signaling pathway. Although the application of $\mathrm{CO}$ to therapeutic modalities are likely to require additional preclinical and clinical studies on its safety and efficacy for human use, strategies for the attenuation of the HSR-induced inflammatory signaling pathway, as shown in the present study, may expand the currently limited therapeutic options.

\section{Acknowledgements}

The authors thank Dr Reiko Akagi for providing cDNAs for TNF- $\alpha$ and iNOS.

\section{References}

1. Bhatia M and Moochhala S: Role of inflammatory mediators in the pathophysiology of acute respiratory distress syndrome. J Pathol 202: 145-156, 2004.

2. Hudson LD, Milberg JA, Anardi D and Maunder RJ: Clinical risks for development of the acute respiratory distress syndrome. Am J Respir Crit Care Med 151: 293-301, 1995.

3. Kollef MH and Schuster DP: The acute respiratory distress syndrome. N Eng J Med 332: 27-37, 1995.

4. Bernard GR, Artigas A, Brigham KL, et al: The AmericanEuropean Consensus Conference on ARDS. Definitions, mechanisms, relevant outcomes, and clinical trial coordination. Am J Respir Crit Care Med 149: 818-824, 1994. 
5. Foresti R, Bani-Hani MG and Motterlini R: Use of carbon monoxide as a therapeutic agent: promises and challenges. Intensive Care Med 34: 649-658, 2008.

6. Otterbein LE, Mantell LL and Choi AM: Carbon monoxide provides protection against hyperoxic lung injury. Am J Physio 276: L688-L694, 1999.

7. Sarady JK, Zuckerbraum BS, Bilban M, et al: Carbon monoxide protection against endotoxic shock involves reciprocal effects on iNOS in the lung and liver. FASEB J 18: 854-856, 2004.

8. Zhang X, Shan P, Otterbein LE, et al: Carbon monoxide inhibition of apoptosis during ischemia-reperfusion lung injury is dependent on the p38 mitogen-activated protein kinase pathway and involves caspase 3. J Biol Chem 278: 1248-1258, 2003.

9. Zuckerbraun BS, McCloskey CA, Gallo D, Liu F, Ifedigbo E, Otterbein LE and Billiar TR: Carbon monoxide prevents multiple organ injury in a model of hemorrhagic shock and resuscitation. Shock 23: 527-532, 2005 .

10. Kanagawa F, Takahashi T, Inoue K, et al: Protective effect of carbon monoxide inhalation on lung injury following hemorrhagic shock/resuscitation in rats. J Trauma 69: 185-194, 2010.

11. Kikenny C, Browne WJ, Cuthill IC, Emerson $M$ and Aitman DG: Improving bioscience research reporting: The ARRIVE guidelines for reporting animal research. J Pharmacol Pharmacother 1: 94-99, 2010

12. Inoue $\mathrm{K}$, Takahashi T, Uehara K, et al: Protective role of heme oxygenase 1 in the intestinal tissue injury in hemorrhagic shock in rats. Shock 29: 252-261, 2008.

13. Murakami K, McGuire R, Cox RA, et al: Heparin nebulization attenuates acute lung injury in sepsis following smoke inhalation in sheep. Shock 18: 236-241, 2002

14. Zegdi R, Fabre O, Cambillau M, et al: Exhaled nitric oxide and acute lung injury in a rat model of extracorporeal circulation. Shock 20: 569-574, 2003.

15. Jiang H, Meng F, Li W, Tong L, Qiao H and Sun X: Splenectomy ameliorates acute multiple organ damage induced by liver warm ischemia reperfusion in rats. Surgery 141: 32-40, 2007.

16. Umeda $\mathrm{K}$, Takahashi $\mathrm{T}$, Inoue $\mathrm{K}$, et al: Prevention of hemorrhagic shock-induced intestinal tissue injury by glutamine via heme oxygenase-1 induction. Shock 31: 40-49, 2009.

17. Maeshima K, Takahashi T, Uehara K, et al: Prevention of hemorrhagic shock-induced lung injury by heme arginate treatment in rats. Biochem Pharmacol 69: 1667-1680, 2005.

18. Sunil VR, Patel KJ, Nilsen-Hamilton M, Heck DE, Laskin JD and Laskin DL: Acute endotoxiemia is associated with upregulation of lipocalin 24p3/Lcn2 in lung and liver. Exp Mol Patho 83: 177-187, 2007.

19. Faleo G, Neto JS, Kohmoto J, et al: Carbon monoxide ameliorates renal cold ischemia-reperfusion injury with an upregulation of vascular endothelial growth factor by activation of hypoxiainducible factor. Transplantation 85: 1833-1840, 2008

20. Ciesla DJ, Moore EE, Johnson JL, Burch JM, Cothren CC and Sauaia A: The role of the lung in postinjury multiple organ failure. Surgery 138: 749-757, 2005.

21. Kohmoto J, Nakao A, Stolz DB, et al: Carbon monoxide protects rat lung transplants from ischemia-reperfusion injury via a mechanism involving p38 MAPK pathway. Am J Transplant 7 : 2279-2290, 2007.

22. Song R, Kubo M, Morse D, et al: Carbon monoxide induces cytoprotection in rat orthotopic lung transplantation via anti-inflammatory and anti-apoptotic effects. Am J Pathol 163: 231-242, 2003.
23. Zuckerbraun BS, Chin BY, Wegiel B, et al: Carbon monoxide reverses established pulmonary hypertension. J Exp Med 203: 2109-2119, 2006

24. Nemzek JA, Fry C and Abatan O: Low-dose carbon monoxide treatment attenuates early pulmonary neutrophil recruitment following acid aspiration. Am J Physiol Lung Cell Mol Physiol 294: L644-L653, 2008.

25. Goebel U, Siepe M, Schwer CI, et al: Postconditioning of the lungs with inhaled carbon monoxide following cardiopulmonary bypass in pigs. Anesth Analg 112: 282-291, 2011.

26. Otterbein LE, Bach FH, Alam J, et al: Carbon monoxide has antiinflammatory effects involving the mitogen-activated protein kinase pathway. Nat Med 6: 422-428, 2006

27. Sarady JK, Otterbein SL, Liu F, Otterbein LE and Choi AM: Carbon monoxide modulates endotoxin-induced production of granulocyte macrophage colony-stimulating factor in macrophages. Am J Respir Cell Mol Biol 27: 739-745, 2002.

28. Morse D, Pischke SE, Zhou Z, et al: Suppression of inflammatory cytokine production by carbon monoxide involves the JNK pathway and AP-1. J Biol Chem 278: 36993-36998, 2003.

29. Nakahira K, Kim HP, Geng XH, et al: Carbon monoxide differentially inhibits TLR signaling pathways by regulating ROS-induced trafficking of TLRs to lipid rafts. J Exp Med 203: 2377-2389, 2006

30. Bilban M, Bach FH, Otterbein SL, et al: Carbon monoxide orchestrates a protective response through PPARgamma. Immunity 24: 601-610, 2006.

31. Hoetzel A, Dolinary T, Vallbracht S, et al: Carbon monoxide protects against ventilator-induced via PPAR-gamma and inhibition of Egr-1. Am J Respir Crit Care Med 177: 1223-1232, 2008.

32. Abdelrahman M, Collin M and Thiemermann C: The peroxisome proliferator-activated receptor-gamma ligand 15-deoxyDelta 12,24 prostaglandin $\mathrm{J} 2$ reduces the organ injury in hemorrhagic shock. Shock 22: 555-561, 2004.

33. Chima RS, Hake PW, Piraino G, Mangeshkar P, Denenberg A and Zingarelli B: Ciglitazone ameliorates lung inflammation by modulating the increased inhibitor kappaB protein kinase/nuclear factor-kappaB pathway following hemorrhagic shock. Crit Care Med 36: 2849-2857, 2008

34. Chima RS, Hake PW, Piraino G, Mangeshkar P, O'Connor M and Zingarelli B: Ciglitazone, a novel inhibitor of lung apoptosis following hemorrhagic shock. Int J Clin Exp Med 3: 1-9, 2010.

35. Rodkey FL, O'Neal JD and Collison HA: Oxygen and carbon monoxide equilibria of human adult hemoglobin at atmospheric and elevated pressure. Blood 33: 57-65, 1969.

36. Morse D, Sethi J and Choi AM: Carbon monoxide-dependent signaling. Crit Care Med 30: S12-S17, 2001.

37. Mayr FB, Spiel A, Leitner J, et al: Effects of carbon monoxide inhalation during experimental endotoxemia in humans. Am J Respir Crit Care Med 171: 354-360, 2005.

38. Brittain T, Sutherland J and Greenwood C: A study of the kinetics of the reaction of ligands with the liganded states of mouse embryonic haemoglobins. Biochem J 234: 151-155, 1986.

39. Wilson MR, O'Dea KP, Dorr AD, Yamamoto H, Goddard ME and Takata M: Efficacy and safety of inhaled carbon monoxide during pulmonary inflammation in mice. PLoS One 5: e11565, 2010. 